\title{
IDENTIFICAÇÃO DE INTERPOLADORES ADEQUADOS A DADOS DE CHUVA A PARTIR DE PARÂMETROS ESTATÍSTICOS
}

\author{
LUCAS, Taíza P. B. - taizaead@gmail.com \\ Doutoranda em Geografia - Programa de Pós-graduação - IGC/UFMG \\ PLEC, Denis - denis.plec@gmail.com \\ Matemático Aplicado e Mestre em Recursos Hídricos - DESA/UFMG \\ ABREU, Magda. L. - magdacrs@ufmg.br \\ Professora Aposentada do Departamento de Geografia - IGC/UFMG \\ PARIZZI, Maria Giovanna - mgparizzi@msn.com \\ Professora do Departamento de Geologia - IGC/UFMG
}

\begin{abstract}
RESUMO: O objetivo desse estudo é definir o interpolador que melhor represente a espacialidade das chuvas em Belo Horizonte para 3 períodos de tempo, para a chuva acumulada mensal, para o total diário e eventos diários de maior intensidade $(\mathrm{mm} / \mathrm{h})$, a partir de 14 pluviômetros de báscula instalados em nove regionais administrativas. A fim de um melhor entendimento dos eventos de chuva e sua variabilidade utilizaram-se técnicas de geoestatísticas para identificação da sua distribuição espacial. Comparou-se os métodos de interpolação por IDW, RBF e KRG através de tratamentos estatísticos dos resíduos gerados pelo método de validação cruzada. Os dados observados e calculados foram comparados a partir da média, desvio padrão, erro médio quadrático (REMQ), correlação $(r)$, de concordância $(d)$ e o índice de confiança $(c)$, gerando uma qualificação do desempenho de ótimo a péssimo para os diferentes interpoladores. A correlação entre os valores indica o grau de dispersão dos dados obtidos em relação à média. O índice $d$ indica o grau de afastamento dos valores estimados em relação aos observados. Seus valores variam de zero, para nenhuma concordância, a 1, para a concordância perfeita. Os resultados obtidos mostram que o método $K R G$ foi o interpolador com os melhores parâmetros estatísticos para chuvas mensais e eventos diários de maior intensidade. Os totais diários apresentaram melhores parâmetros através do método $R B F$, apesar da análise visual do mapa apontar para melhor suavização das isoietas por $K R G$.

Palavras-chave: chuvas, interpoladores e parâmetros estatísticos.

IDENTIFICATION OF INTERPOLATOR PROPER TO RAIN DATA FROM STATISTICAL PARAMETERS

ABSTRACT: The aim of this study is to define the interpolator that best represents rains spatiality in Belo Horizonte for 3 periods of time, for the accumulated monthly rainfall, for the daily sum and daily events of greatest intensity $(\mathrm{mm} / \mathrm{h})$, from 14 rain gauges of weighbridge installed in nine administrative regions. In order to better understand the precipitation events and its variability, geostatistical techniques were used for their spatial distribution identification. The methods of interpolation were compared by IDW, RBF and KRG through statistical treatments of waste generated by cross validation method. The observed and calculated and calculated data were compared from the mean, standard deviation, mean square error (REMQ), correlation ( $r$ ) of agreement (d) and the confidence index (c), generating a qualifying performance from great to poor for various interpolators. The correlation between the values indicates the dispersion degree of the data obtained from the average. The index $d$ indicates the deviation degree of the estimated values in relation to those observed. It values range from zero, for no agreement, to 1 , for perfect agreement. The results show that the KRG method was the interpolator with the best statistical parameters for monthly rainfall and greatest intensity daily events. The daily sums showed better parameters through the RBF method, despite the map visual analysis points to better smoothing of isohyets by KRG.
\end{abstract}

Keywords: rain, interpolators and statistical parameters.

\section{INTRODUÇÃO}

O desenvolvimento do SIG (Sistemas de Informação Geográfica) e o aprimoramento das técnicas de geoprocessamento possibilitam a aplicação de geoestatística à modelagem de dados espaciais, permitindo transformar dados discretos em contínuos pôr meio de interpolação matemática aos dados pontuais, de área e de superfície. Os interpoladores são ferramentas matemáticas que atribuem valores relativos a alguma variável a pontos inseridos em um campo de valores já existentes ou banco de dados construídos a partir de observações sistemáticas e georreferenciadas. 
Os recentes estudos de espacialização de dados climáticos tem salientado a importância de definir os melhores interpoladores. Segundo Aranoff (1989), a qualidade de uma interpolação depende da distribuição e do conhecimento dos pontos usados no cálculo, além da correlação de modelos estatísticos com os fenômenos em estudo. A escolha de um modelo apropriado é essencial para se obter resultados aceitáveis.

A integração entre modelos matemáticos e técnicas de geoprocessamento é importante por possibilitar quantificar a qualidade dos modelos de superfície através da medição do erro estatístico estimado para superfícies previstas. Segundo Silva, J. et. al. (2011), a obtenção de superfícies interpoladas envolve três etapas fundamentais, a análise estatística exploratória de dados espaciais, a análise estrutural de cálculo (modelagem das propriedades superficiais de locais próximos, cálculo e modelação do variograma) e a avaliação dos resultados.

A espacialização dos tipos de chuva requer a escolha do melhor método de interpolação, para que seja possível entender a variabilidade espacial de eventos com diferentes durações e acumulados de chuva e identificar possíveis características espaciais. Segundo Andriotti (2003), a qualidade essencial de uma estimativa não é simplesmente associar um valor a um ponto ou a um bloco, mas associar a essa avaliação a ideia de qualidade da estimativa, dimensionando o erro existente entre o valor atribuído (estimado) por uma modelagem matemática e o valor observado.

O objetivo desse estudo é definir o interpolador que melhor represente a espacialidade das chuvas em Belo Horizonte, tanto do acumulado mensal, como do total diário e a maior intensidade $(\mathrm{mm} / \mathrm{h})$ ocorrida em Novembro de 2011. O acumulado mensal apresenta uma menor variabilidade espacial, enquanto os eventos de pequena duração e grande intensidade apresentam maior variabilidade. Assim é possível verificar o melhor desempenho matemático dos interpoladores em diferentes distribuições temporais para a área de estudo, que irá colaborar para a representação da distribuição espacial de diferentes tipos de chuvas.

Em um ambiente urbano, densamente ocupado, é necessário o entendimento da distribuição da chuva numa escala cartográfica grande, para que os efeitos à população possam ser evitados através de um planejamento eficaz do sistema de drenagem urbana. A grande maioria dos estudos sobre espacialização da chuva se dá a partir de interpolação de grandes áreas, como observado em Reis et. al. (2005) ao espacializar dados de precipitação para projetos de drenagem no Estado de Goiás e Amorim et. al. (2006) para avaliação de dois métodos de espacialização da precipitação pluvial para o Estado de Alagoas.

Segundo Tao, T. et. al. (2009), a natureza estocástica da chuva dificulta a modelagem da variável, que apresenta incertezas desde a medição, transmissão e erros na escolha da interpolação. A distribuição da precipitação pode ser influenciada pelo tipo de solo, variabilidade da topografia, assim como o efeito de sombreamento das vertentes. Para os autores, é complicado estimar com precisão a distribuição espacial das chuvas, assim como extrapolação de medições pontuais sobre grandes áreas.

Uma forma de melhorar a estimativa da precipitação é aumentar o número de postos pluviométricos de uma rede de monitoramento. Tradicionalmente, os postos são escassos e compreendem extensas áreas. Devido a essa baixa densidade de dados muitos autores utilizam redes de monitoramento de diferentes áreas para minimizar a baixa densidade das estações no local estudado. Porém, muitas vezes eles têm metodologias diferentes de coleta de dados, onde algumas não seguem o padrão internacional, o que diminui a confiabilidade dos produtos gerados através de métodos de interpolação.

Tao, T. et. al. (2009) utilizaram como base de dados 30 pluviômetros de báscula, para espacialização da chuva em Lyon, numa área de $460 \mathrm{Km}^{2}$, ou seja, um pluviômetro para 
cada $15 \mathrm{Km}^{2}$. Em Belo Horizonte, tem-se 14 pluviômetros de báscula da rede URBELUFMG (Companhia Urbanizadora de Belo Horizonte em parceria com a Universidade Federal de Minas Gerais) e 2 da rede de estações automáticas do INMET (Instituto Nacional de Meteorologia). Segundo o IBGE (2010), a capital mineira tem 331,4 $\mathrm{Km}^{2}$, o que corresponde a uma densidade de um pluviômetro para cada $20,7 \mathrm{Km}^{2}$. A distribuição não é homogênea, a maioria apresenta-se alinhada a Serra do Curral, no sentido SW-NE, porém há pelo menos uma estação em cada uma das nove regionais administrativas da Prefeitura de Belo Horizonte - MG (PBH-MG), como ilustra a figura 1.

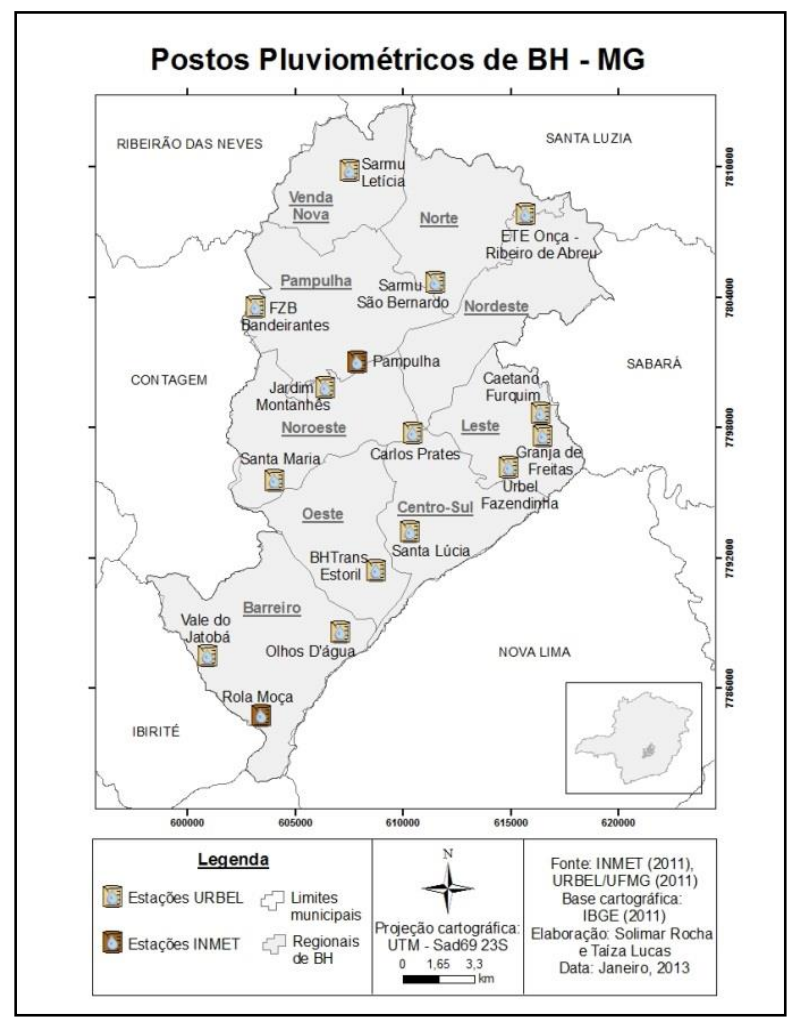

Figura 1: Localização dos Postos Pluviométricos em Belo Horizonte-MG.

\section{MATERIAIS E MÉTODOS}

A rede de monitoramento implantada pela URBEL-UFMG foi instalada em 2008, contendo 12 pluviógrafos com registro de chuva a cada 15 min e 2 pluviógrafos com registro a cada $5 \mathrm{~min}$. As estações automáticas do INMET são de 2006 (estação automática da Pampulha) e de 2008 (estação automática do Parque do Rola Moça), fornecendo dados a cada hora.

A chuva foi analisada a partir da intensidade $(\mathrm{mm} / \mathrm{h})$, sendo feito o somatório a cada hora nas estações da rede URBEL-UFMG, e comparadas à registrada no mesmo intervalo nas estações automáticas do INMET. A chuva diária foi manipulada segundo a metodologia do INMET, com o somatório de 13 as 12 UTC do dia de registro. A chuva mensal foi calculada como o somatório deste registro diário para cada estação.

A partir da ferramenta geoestatística do software ArcGIS 9.3 (ESRI, 2003) foi utilizado os modelos matemáticos de interpolação determinísticos, como o Inverse Distance Weighting (IDW) e o Radial Basis Functions (RBF) e geoestatísticos, como a Krigagem (KRG). Segundo Marcuzzo et. al. (2011), a interpolação por IDW determina os valores nos pontos interpolados usando uma combinação linear ponderada dos pontos amostrados. O peso de cada ponto é o inverso de uma função da distância. É um método computacionalmente rápido, já que a definição dos pesos para a ponderação linear é feito de uma maneira simplista. Nesse método os pontos de dados estão no interior das isolinhas equivalentes, porém geralmente há a geração de muitas "ilhas" de dados e as isolinhas não são muito suaves. Moura (2012) ressalta que o IDW é indicado quando o 
interesse é a área de influência dos valores e pontos, resultando em um valor em cada ponto do território.

O interpolador RBF é um modelo determinístico, assim como o IDW, porém mais flexível. Também não oferece ferramentas de correlação entre os resíduos e os dados estimados. Métodos RBF são uma forma de redes neurais artificiais, com cinco funções de base diferentes: thin-plate spline, spline with tension, completely regularized spline, multiquadric function e inverse multiquadric spline (ESRI, 2003). Cada função de base tem uma forma e resulta numa superfície de interpolação suavizada. Ao comparar $R B F$ com o método IDW, o RBF irá prever valores acima ou abaixo do valor observado, seguindo a tendência de suavização da superfície.

Segundo Wanderley et al. (2012), a Krigagem (KRG) se destaca de outros métodos por possibilitar a análise da variância dos dados através da análise variográfica, o que demonstra a descontinuidade espacial da amostra. A KRG pode ser entendida como uma série de técnicas de análise de regressão que procura minimizar a variância estimada a partir de um modelo prévio, que leva em consideração a dependência estocástica dos dados distribuídos no espaço georreferenciado.

Para estimativa do erro, utilizou-se da validação cruzada dos dados em todos os interpoladores, através da metodologia apresentada em Andriott (2003), na qual um posto específico é descartado sucessivamente na realização da interpolação. Assim, é possível obter o valor estimado $(E)$, relativo ao posto retirado e, posteriormente, compará-lo com o valor real da variável $(O)$ e identificar o erro da estimativa $(E-O)$. $O$ que permitiu a montagem das planilhas com os valores estimados (E) e observados (O) e o erro percentual em cada ponto de coleta, para cada interpolador.

\section{1) Análise Estatística Exploratória}

A análise estatística exploratória foi realizada para os dados de Novembro de 2011 com o uso do software SPSS 13.0, para os principais parâmetros estatísticos, tanto de tendência central como medidas de dispersão, no intuito de verificar o padrão de distribuição espacial dos dados. A partir dos parâmetros apresentados por Andriotti (2003), baseados no coeficiente de variação (CV), na assimetria e na curtose, decidiu-se realizar a transformação logarítmica para um melhor ajuste.

As medidas de dispersão permitem identificar as diferenças entre amostras com as mesmas medidas de tendência central. Assimetria é o grau de afastamento de uma curva no sentido horizontal, ou seja, o grau de afastamento da média em relação à moda e mediana correspondente. Curtose é o grau de achatamento de uma curva em relação a uma curva representativa de uma distribuição normal que possui uma assimetria igual a zero e curtose igual a três. A curva está bem ajustada ao modelo normal se tiver assimetria entre $\pm 3[\sqrt{ }(6 / n)]$ e curtose entre $\pm 6[\sqrt{ }(6 / n)]$ (ANDRIOTTI, 2003).

Segundo o autor, CV é o resultado do desvio padrão divido pela média, e permite uma ideia de regularidade ou homogeneidade da amostra, multiplicado por cem, indica o percentual de afastamento da média. Quando o CV for maior que um, aconselha-se a transformação logarítmica dos dados. Esta transformação é um caso especial de BoxCox, quando $\lambda=0$, a transformação é a seguinte: $y(s)=\ln (Z(s))$, de $Z(s)>0$, e In é o logaritmo natural. Ela é frequentemente utilizada quando os dados tem uma distribuição assimétrica positiva (média > mediana) e existem alguns valores muito grandes (ESRI, 2003), como identificado nas amostras analisadas.

Os métodos de interpolação apresentam melhor eficácia, resíduos menores, quando os dados possuem distribuição normal. Os gráficos de histograma e Quantil-Quantil Plot (QQPlot) permitem explorar os efeitos de diferentes transformações na distribuição do conjunto de dados. O gráfico QQPlot é muito utilizado para determinar se dois conjuntos de dados pertencem à mesma distribuição de probabilidades. Em tal 
gráfico, os pontos são formados pelos quantis amostrais e se na análise os pontos alinham-se numa reta de inclinação um, as distribuições de probabilidade das duas amostras podem ser consideradas as mesmas. O gráfico QQPlot permite compreender quais são os valores que afastam da reta de inclinação 1 e caracterizam-se como outlier (destoante da amostra).

\section{2) Modelagem Espacial}

A aplicação de um modelo de interpolação requer uma análise da tendência direcional dos dados espaciais da superfície. A técnica Trend Surface Analysis é uma representação de variáveis numéricas a partir de uma regressão polinomial. Por meio de uma superfície gerada por uma polinomial através do método dos mínimos quadrados, separam-se os dados em duas componentes, uma regionalizada e outra localizada. Segundo Andriotti (2003), a remoção ou identificação do Trend isola e/ou enfatiza os componentes locais através dos resíduos. Este procedimento é importante, principalmente se os valores em análise se ajustarem a uma distribuição normal. As superfícies geradas são muito sensíveis à presença de outliers, que devem ser removidos de acordo com o entendimento da natureza da variável e dos objetivos estudados.

O ajuste do Trend Surface Analysis é importante para definir o grau que deve ter a superfície polinomial para melhor se adequar aos dados disponíveis. A técnica foi feita com o uso do software ArcGIS (2003), ferramenta geoestatística, a partir da análise de tendência fornecida numa perspectiva tridimensional. A localização dos pontos de amostragem são plotados no plano ( $x, y)$, acima de cada ponto da amostra, o valor é dado pela altura na dimensão $z$. Uma característica da ferramenta de análise de tendências é que os valores são projetados no plano $(x, z)$, e $(y, z)$ como gráficos de dispersão.

O variograma é a ferramenta básica para o procedimento de interpolação através do método de $K R G$, pois o mesmo permite uma representação quantitativa da variação de um fenômeno regionalizado no espaço (Carvalho et al., 2009). Andriotti (2003) sugere analisar o semivariograma em pelo menos quatro direções, quando realizado em apenas duas corre-se o risco de não caracterizar a anisotropia, ou seja, quando os semivariogramas mostram distintos comportamentos para diferentes direções de linhas de amostragem. A isotropia ocorre quando a auto-correlação espacial depende apenas da distância entre dois pontos, sem influência do fator direcional. Identificou-se, nos dados de chuva de novembro de 2011, anisotropia geométrica, ou seja, o alcance varia de acordo com as diversas direções, permanecendo constante o patamar, os melhores parâmetros de correlação foram influenciados pelo sentido das tendências dos dados (LANDIM, 2006).

Os modelos de variograma podem ser ajustados a modelos teóricos. Segundo Andriotti (2002), este ajuste é feito de modo interativo, testando-se os parâmetros do variograma (efeito pepita, alcance, patamar e número de estruturas presentes) até que se considere um modelo satisfatório. Para tanto, utilizou-se os valores calculados automaticamente e ajustados após o cálculo da média da distância dos vizinhos mais próximos.

A análise geoestatística é realizada com o objetivo de determinar o modelo de variabilidade espacial dos dados, utilizado na estimativa pelo método da $K R G$. Segundo Andriotti (2003), a KRG ordinária é muito utilizada nos estudos de geociências por apresentar um crescimento rápido na origem, onde tem um comportamento linear. Segundo Silva et. al. (2011), por defeito, estes estimadores apresentam uma variância mínima e a média relativamente constante, mas desconhecida (estacionaridade). 0 modelo de $K R G$ ordinária leva em consideração as variações locais da variável, limitando o seu domínio de estacionaridade a uma vizinhança local. No ajuste do modelo é necessário determinar os pesos da $K R G$, que dependem da localização do ponto, numero de vizinhos e configuração geométrica entre as amostras. 
A validação cruzada permite inferir sobre o melhor interpolador e sobre os diferentes modelos de KRG. A partir da análise estatística dos resíduos e da observação do gráfico de regressão, entre os valores estimados e os observados, determina-se o interpolador mais adequado. Se a validação cruzada apresentar os resultados sobre uma reta de regressão próxima da bissetriz e com pequena dispersão dos pontos em torno dessa reta, tem-se uma boa estimativa (ANDRIOTTI, 2003). Os gráficos de regressão linear foram gerados no pacote office Excel do Windows 2007 e avaliados simultaneamente aos parâmetros estatísticos.

\section{3) Métodos Estatísticos de Comparação}

Os dados observados no mês de novembro de 2011, totais diários dos dias 15 e 27 e os maiores totais horários, em Belo Horizonte, foram correlacionados com os estimados através de regressão linear. Como critério de decisão do melhor interpolador, foram utilizados os seguintes índices estatísticos: média dos resíduos, desvio padrão dos resíduos, erro médio quadrático $(R E M Q)$, correlação $(r)$, concordância $(d)$ e o índice de confiança (c). Um dos critérios de acurácia do interpolador é a normalidade dos resíduos, portanto espera-se que a média tenha um valor próximo de zero e a raiz quadrada da variância igual a um (ROGERSON, 2010).

O REMQ é descrito segundo a equação 1, proposto por Legates \& Mccabe Jr. (1999):

$$
\text { REQM }=\sqrt{\frac{\sum_{j=1}^{J}\left(\mathrm{O}_{i}-E_{i}\right)^{2}}{J}}
$$

Em que: J - número de observações, O - valor observado experimentalmente e E - valor estimado pelo método.

A avaliação do desempenho dos métodos foi baseada nos coeficientes de correlação " $r$ " (entre o valor estimado e o observado), de concordância " $d$ " de Willmott e de confiança " $C$ ", produto dos índices anteriores, proposto por Camargo e Sentelhas (1997). A precisão é dada pelo coeficiente de correlação que indica o grau de dispersão dos dados obtidos em relação à média, ou seja, o erro aleatório. A exatidão está relacionada ao afastamento dos valores estimados em relação aos observados. Matematicamente essa aproximação é dada por um índice designado de concordância, representado pela letra " $d$ " (WILLMOTT et. al., 1985). Seus valores variam de zero, para nenhuma concordância, a 1, para a concordância perfeita. $O$ índice é dado pela equação 2 :

$d=1-\frac{\sum_{i=1}^{i=n}\left(E_{i}-O_{i}\right)^{2}}{\sum_{i=1}^{i=n}\left(\left|E_{i}-\bar{O}\right|+\left|O_{i}-\bar{O}\right|\right)^{2}}$

Em que: $\mathrm{E}$ - valor estimado, $\mathrm{O}_{\mathrm{i}}$ - valor observado e $\bar{O}$ - a média dos valores observados.

Camargo e Sentelhas (1997) propuseram uma qualificação dos interpoladores a partir do produto entre os valores de $r$ e $d$, como apresentado na tabela 1 :

Tabela 1. Análise do desempenho do modelo com base no índice de confiança

\begin{tabular}{|c|c|}
\hline Valor de $c$ & Desempenho \\
\hline$>0,85$ & Ótimo \\
\hline 0,76 a 0,85 & Muito bom \\
\hline 0,66 a 0,75 & Bom \\
\hline 0,61 a 0,65 & Mediano \\
\hline 0,51 a 0,60 & Sofrível \\
\hline 0,41 a 0,50 & Mau \\
\hline$<0,40$ & Péssimo \\
\hline
\end{tabular}

Fonte: Camargo \& Sentenlhas (1997) 


\section{RESULTADOS}

1) Análise Exploratória dos Dados

Os totais mensais das 16 estações pluviométricas de Belo Horizonte apresentaram uma distribuição assimétrica, duas estações apresentaram dados muito inferiores às demais, tanto diário como mensal, o que provavelmente indica problemas de manutenção dos equipamentos ou no processo de transmissão, através do sistema Datalogger. As estações, Vale dos Olhos D'água e Jardim Montanhês, foram descartadas da análise devido à ausência de dados na maioria dos dias de novembro de 2011.

A Tabela 2 mostra os parâmetros estatísticos dos totais mensais, do total diário e máxima intensidade dos dias 15/11/11 e 27/11/11 das 14 estações utilizadas. Optou-se por manter a análise desses dois dias, pois as maiores intensidades ocorreram em ambos, sendo que no dia 15/11 ocorreu uma chuva melhor distribuída no tempo e espaço belo-horizontino e no dia $27 / 11$ mais concentrada espacialmente.

A partir da Tabela 2 observa-se que os dados mensais são os que apresentam melhor distribuição. A partir do CV observa-se que o desvio padrão está a $19 \%$ da média. Tanto o total diário, como a intensidade, do dia 15/11 foi melhor distribuído que o dia 27/11, enquanto que o total do dia $15 / 11$ está $21 \%$, o total diário do dia $27 / 11$ está a $35 \%$, a maior intensidade ocorrida no dia 15 está a $36 \%$ e do dia $27 / 11$ a $69 \%$. A partir do parâmetro de ajuste, a curva normal através da curtose e assimetria, identificou-se que o valor de curtose $( \pm 3,92)$ está acima do padrão de ajuste para a chuva horária do dia $27 / 11$, e todas as amostras estiveram dentro do padrão para a assimetria $( \pm 1,95)$.

Tabela 2 - Análise estatística dos dados de chuva de Novembro de 2011

\begin{tabular}{|c|c|c|c|c|c|}
\hline & Mensal & $\begin{array}{l}\text { Diária } \\
15 / 11\end{array}$ & $\begin{array}{l}\text { Diária } \\
27 / 11\end{array}$ & $\begin{array}{c}\text { Horária } \\
15 / 11\end{array}$ & $\begin{array}{c}\text { Horária } \\
27 / 11\end{array}$ \\
\hline $\mathrm{N}$ & 14 & 14 & 14 & 14 & 14 \\
\hline Valor Min & 215 & 28,6 & 36,6 & 10,2 & 4 \\
\hline Valor Max & 380,8 & 58 & 104,6 & 31,2 & 30,6 \\
\hline Média & 264,81 & 40,29 & 57,98 & 18,24 & 10,43 \\
\hline Des. Padrão & 49,29 & 8,14 & 19,28 & 6,24 & 7,10 \\
\hline Assimetria & 0,993 & 0,549 & 1,183 & 0,655 & 1,734 \\
\hline Curtose & 3,243 & 2,682 & 3,644 & 2,293 & 5,669 \\
\hline $\mathrm{CV}$ & 0,186 & 0,213 & 0,348 & 0,357 & 0,695 \\
\hline $1^{\circ}$ Quartil & 221,4 & 34,2 & 42,2 & 13 & 5,2 \\
\hline Mediana & 261,4 & 38,9 & 53,5 & 15,7 & 8,4 \\
\hline $3^{\circ}$ Quartil & 284,4 & 46,2 & 66,6 & 23,6 & 11,6 \\
\hline
\end{tabular}

Fonte de dados: URBEL/UFMG e INMET.

O gráfico de histograma permite compreender a frequência de casos por classes e a distribuição do fenômeno. Os gráficos produzidos ressaltaram informações consistentes com a tabela 2. Para os dados mensais, a primeira classe, entre 200 e $215 \mathrm{~mm}$, teve 4 estações, a segunda com maior número de estações foi entre 250 e $300 \mathrm{~mm}$, onde se encontra a média. O histograma de dados diários do dia 15/11/11 apresentou 4 classes com 3 estações, entre elas o valor médio, o que ressalta a distribuição homogênea dos dados. Os dados diários do dia 27/11/11 teve maior número de elementos entre 50 a 60 $\mathrm{mm}, 5$ estações das 14, as classes de intervalo menores somaram 5 e as classes de intervalo maiores somaram 4. O histograma da máxima intensidade do dia 15 apresentou a primeira classe com 6 elementos, duas classes com 3 estações cada e mais duas classes com 1 elemento. A máxima intensidade do dia 27 apresentou uma distribuição assimétrica, a segunda classe, entre 5 e $10 \mathrm{~mm}$, constitui-se de 6 estações, a primeira classe teve 3 , a terceira dois elementos e a ultima classe, apenas uma estação, que registrou $30,6 \mathrm{~mm} / \mathrm{h}$.

Os gráficos de 1 a 5 demostram a distribuição ao longo de uma reta de inclinação um e as estações que apresentam alguma anormalidade, como outlier, a partir de gráficos tipo 
QQPlot, elaborados no software SPSS 13 . O gráfico 1 representa a distribuição mensal, nota-se que o valor mais afastado da reta é a estação 14, estação automática do INMET situada no Parque do Rola Moça, a 1200 m de altitude. Seguido da estação 1, Vale do Jatobá, a $955 \mathrm{~m}$, ambas situadas na Regional Barreiro, na borda S/SW da Serra do Curral. A estação 6, que também se encontra afastada da reta, localizada no extremo oeste da Regional Pampulha, na Fundação Zoobotânica da PBH, foi o menor registro mensal, porém não consideravelmente destoante das estações vizinhas. Das cinco estações de menor registro, quatro estão situadas no quadrante oeste/norte do município.

O gráfico 2 representa a distribuição do total diário do dia 15/11/11. Observa-se que os dados estão bem ajustados a reta, com uma menor variância em relação as demais amostras. A estação 11, BHTrans - Estoril, situada na Regional Oeste, a 959 m de altitude, registrou o valor mais alto, acúmulo diário de $58 \mathrm{~mm}$. O único que se encontra afastado da reta, caracterizado como outlier. O ajustamento a curva de inclinação 1 , se deve a homogeneidade dos dados, verificada tanto na Tabela 2, como relatado sobre o gráfico de histograma.

No dia 27/11/11, gráfico 3, três estações não estão bem ajustadas à curva, sendo: Vale do Jatobá (1), Parque do Rola Moça (14) e na unidade de apoio da URBEL - Fazendinha (10), no complexo de favelas do Aglomerado da Serra, a uma altitude de $919 \mathrm{~m}$, na vertente oeste da Serra do Curral, na Regional Centro-Sul. As duas primeiras apresentaram acúmulos elevados, 104,6 e $88,4 \mathrm{~mm}$, respectivamente, enquanto a terceira registrou um total diário de $36,6 \mathrm{~mm}$, não muito inferior às estações vizinhas, Granja de Freitas (12), a 897 m, 42 mm, Caetano Furquim (5), a 804 m, à jusante do Córrego Arrudas, 42,2 mm, ambas na Regional Leste.

O gráfico 4, do maior total horário do dia 15/11, diferentemente do gráfico 2, representa alguns dados afastados da reta. A distribuição dos dados horários se dá de forma assimétrica positiva. As estações mais afastadas da reta de inclinação 1 são: SAMU - São Bernardo (7), na Regional Norte, a estação Santa Maria (2), no extremo oeste, Caetano Furquim (5), na Regional Leste, com acumulado horário de 22,6, 23,6 mm/h e 26,4 $\mathrm{mm} / \mathrm{h}$. As estações com menor acúmulo ocorreram na Fundação Zoo-Botânica da PBH (6) e na estação de Venda Nova (9), no extremo norte, com registro de 10,2 e 12,8 $\mathrm{mm} / \mathrm{h}$, respectivamente. A chuva ocorrida neste período concentrou mais de $40 \%$ da chuva diária, em todas as estações.

O gráfico 5, assim como 3, mostra que as estações mais afastadas da reta são a 1 , Vale do Jatobá, no Barreiro, e a 10, URBEL - Fazendinha, na Regional Centro-Sul. A chuva foi menos concentrada temporalmente, em todas as estações, a maior intensidade corresponde a $20 \%$ do acumulado diário. As Regionais, Pampulha, Noroeste e Barreiro, lado oeste do município registraram muito mais chuva que o leste, tanto diária como horária. Enquanto que a chuva horária que ocorreu no dia 15 , variou de $10 \mathrm{~mm} / \mathrm{h}$ na Pampulha (Fundação Zoo-Botânica da PBH) a 31,2/h mm na Regional Oeste, a chuva horária do dia 27, variou de $4 \mathrm{~mm} / \mathrm{h}$ na Regional Centro-sul a $30,6 \mathrm{~mm} / \mathrm{h}$ no Barreiro.

Os valores mensais, diários e horários do dia 27/11/11, acumulados nas estações a S/SW do município, são outliers na observância estatística, mas não podem ser retirados da análise porque refletem um comportamento da distribuição espacial da chuva em Belo Horizonte, já identificados por autores, como Moreira (2002) e Reis et. al (2004). Ambos trabalharam com o total diário de chuva, identificaram a espacialidade das chuvas durante os meses de setembro a abril e sua relação com a topografia local. Moreira (2002) analisou a espacialidade em toda RMBH e Reis et. al. (2004) agrupou a quantidade de precipitação diária acumulada em quatro categorias, de chuvas fracas a chuvas extremamente fortes, verificando que as chuvas mais intensas ocorrem alinhadas a Serra do Curral. Os dados observados mostram que tanto o acumulado mensal, como o 
total do dia 27/11 e o maior acúmulo horário, 30,6 mm, no Barreiro, ocorreram não alinhada, mas concentrada na porção S/SW, de maior elevação no território municipal.
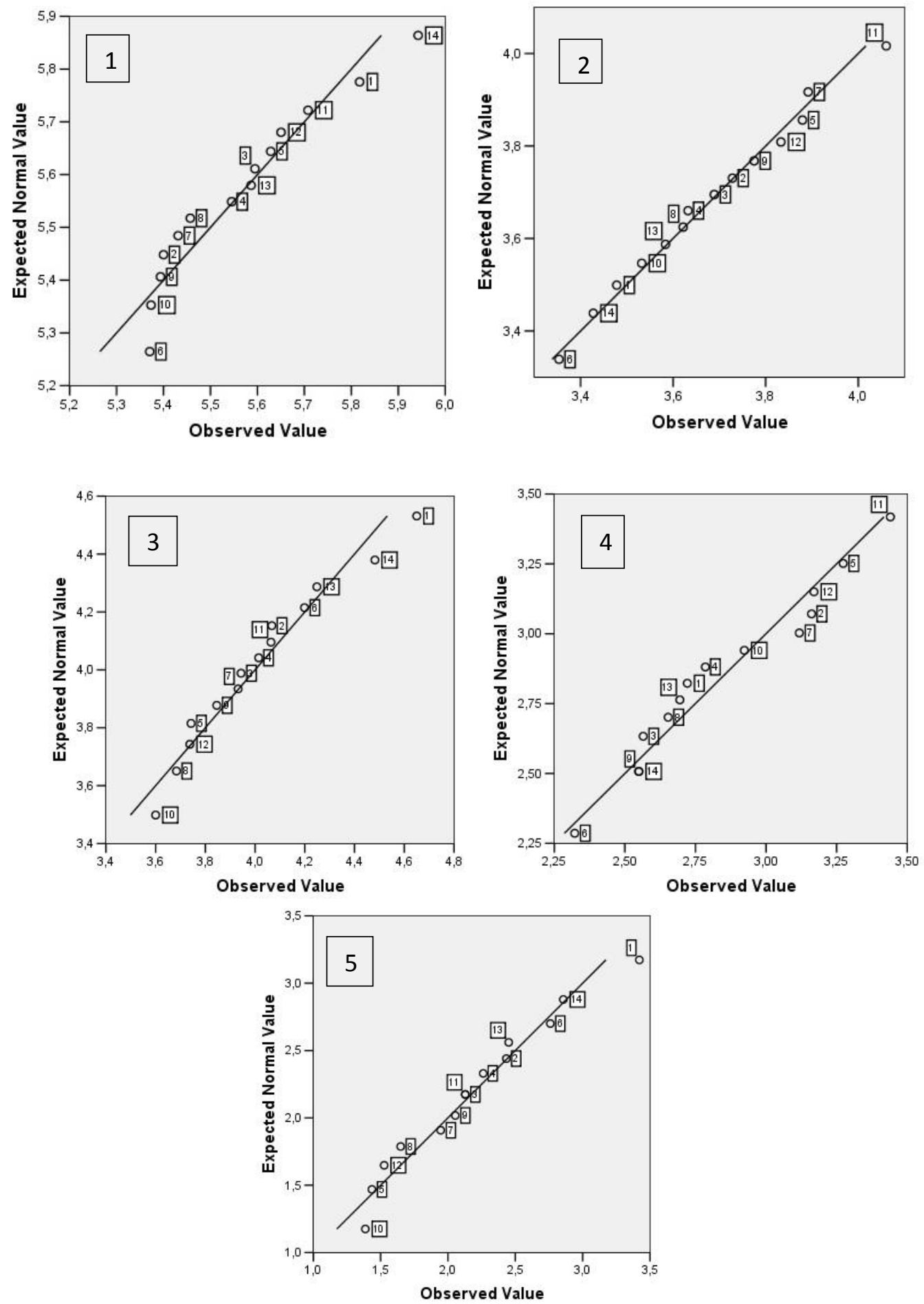

Gráficos 1 a 5: Gráfico QQPlot ajustados à distribuição normal. 1) Dados Mensais de Novembro de 2011, 2) Total Diário de 15/11/11, 3) Total Diário de 27/11/11, 4) Maior intensidade do dia 15/11/11 e 5) Maior intensidade do dia 27/11/11. Fonte de dados: URBEL/UFMG e INMET.

2) Modelagem Espacial 
A partir do Trend Surface Analysis identificou-se que a curva gerada tanto $\mathrm{x}, \mathrm{z}$ e $\mathrm{y}, \mathrm{z}$ tem um padrão polinomial de segunda ordem ajustado à curva. As tendências dos dados mensais são sul e leste, onde os valores foram maiores. Os dados diários do dia 15/11, assim como a maior intensidade deste dia, demostram uma curva polinomial de segunda ordem acentuada, o que indica uma influência mais forte dos dados centrais comparados com os de todas as fronteiras. O dia $27 / 11$, assim como o maior acúmulo horário, tem uma tendência acentuada no sentido sul e oeste, mais inclinada que os dados mensais. Os valores que direcionam o Trend Surface Analysi, novamente, não foram removidos do processo de interpolação por representar uma característica importante da distribuição espacial da chuva em Belo Horizonte, devido a forçante topográfica no sentido meridional de direção SW-NE.

A análise variográfica dos dados mensais de novembro de 2011 mostrou características anisotrópicas geométricas de variograma do tipo com patamar. A distância entre as classes de pares foi dada pela media de vizinhos mais próximos, de aproximadamente 9 $\mathrm{Km}$, e 14 classes de pares de pontos. O gráfico de covariância demonstrou que o raio de influência de um ponto em relação a outro se estende até $7,9 \mathrm{~km}$. Este valor de alcance expressa a zona de influência do campo estruturado (variáveis correlacionadas) e a do campo aleatório (variáveis independentes). Para geração dos mapas seguintes, diários e horários dos dias 15/11/11 e 27/11/11 mantiveram-se os mesmos parâmetros. Ao analisar os semivariogramas gerados pelo modelo, identificou-se que o melhor ajuste é o esférico, a partir da comparação com os modelos teóricos apresentados por Andriotti (2003).

A partir destas características, e analisando visualmente o comportamento de todos os semivariograma de cada amostra, mantiveram-se os mesmos parâmetros acima, sendo retirado da geração da figura 2 , o posto 10, URBEL - Fazendinha, devido ao distanciamento das estações vizinhas, apesar de estar ajustado a curva QQplot, como mostra o gráfico 1. Da fig. 3, foram retiradas as estações Santa Maria, Santa Lucia e São Bernardo, posto 2, 3 e 7, também pelo distanciamento entre os vizinhos mais próximos, ressaltando a tendência global, e pelo distanciamento da reta do gráfico 4 . Ressalta-se que nas estações 2 e 7 , os valores acumulados estão entre as 5 estações de maiores registros e a 3 está entre as 5 estações de menor registro.

3) Métodos Estatísticos de comparação

Para o cálculo dos resíduos foram produzidos 15 mapas, sendo 5 de cada interpolador, $I D W, R B F$ e KRG (Ordinária, modelo teórico de variograma esférico), para cada amostra (mensal, diário dos dias 15 e 27 seus respectivos totais horários). As figuras 2 a 4 mostram todos os mapas importantes na análise visual da distribuição da chuva em Belo Horizonte, assim como diferenciações entre os produtos gerados pelos interpoladores.

A tabela 3 apresenta os índices estatísticos calculados para analisar o desempenho dos interpoladores para a espacialização da precipitação em Belo Horizonte, em diferentes escalas temporais. Os parâmetros utilizados mostram que para a espacialização do total mensal de chuva, o método da KRG Ordinária esférica apresentou a menor média dos resíduos, assim como menor desvio padrão e menor REQM. Maior índice $r, d$ e $c$, classificado como muito bom a partir dos critérios de Camargo e Sentelhas (1997).

A figura 2 mostra que o mapa feito a partir do método da $K R G$ é mais suavizado, enquanto que o mapa gerado por $R B F$ tem uma área maior na segunda classe de dados, já o mapa interpolado por IDW é o menos suavizado, com linhas mais retangulares entre as classes. Dentre os interpoladores, este é o menos satisfatório para dados mensais, tanto através dos parâmetros estatísticos, como a análise visual da imagem. Como identificado na análise do Trend Surface Analysis, o total mensal ocorreu de forma mais concentrada na porção $\mathrm{E} / \mathrm{SE} / \mathrm{S}$, sendo maior acúmulo mensal no extremo $\mathrm{SE}$, onde se situa a maior altitude do município. 
O total diário do dia 15/11, assim como a sua maior intensidade, apresentaram os piores índices. O índice de confiança $c$ foi extremamente baixo em função dos valores de correlação entre os dados observados e os estimados. Apesar do melhor ajustamento a distribuição normal, os parâmetros não mostraram um desempenho satisfatório. 0 método $R B F$ apresentou o valor médio dos resíduos mais próximos de zero, o menor desvio padrão e REQM. A máxima intensidade do 15/11 apresentou a menor média dos resíduos de todas as amostras, acompanhado pelos menores valores de $R E Q M$, dado pelo método da KRG ordinária. Ressalta-se que os coeficientes $r, d$ e $c$ sugerem um desempenho péssimo de todos os interpoladores, devido à baixa correlação entre eles.

Na tentativa de melhorar a interpolação, foram descartadas as estações São Bernardo (7), Santa Maria (2) e Santa Lucia (3), indicados pelo desvio da reta do gráfico 4 e pela distancia entre os pares dado pelo variograma. A figura 3 mostra o mapa gerado por $K R G, R B F$ e IDW para maior acumulado horário do dia 15. Nesta amostra não foi identificado uma tendência espacial, mas a análise criteriosa da distribuição ao longo da reta dada pelo gráfico QQPlot e análise do variograma são fundamentais para o ajustamento do modelo e geração da imagem de representação da distribuição espacial da chuva. Verifica-se nesta figura, que a chuva ocorreu concentrada na porção SE, nas Regionais Oeste e Centro-Sul, nas franjas da Serra do Curral. O mapa interpolado por $R B F$, apesar de não ter os melhores parâmetros estatísticos, nota-se maior suavização nas isoietas. No entanto, observam-se círculos concêntricos em torno de valores menores que os vizinhos mais próximos, assim como o mapa gerado por IDW.

De acordo com o critério definido por Camargo \& Sentelhas (1997), o método RBF foi considerado muito bom para a estimativa dos valores de chuva diária ocorrida no dia $27 / 11 / 11$. O método foi mais eficiente devido à correlação satisfatória entre os valores reais e os valores estimados, em função do menor $R E M Q$ e dos outros índices estatísticos que apontam nesta direção. A figura 4 mostra os mapas interpolados por $K R G, R B F$ e $I D W$, nota-se que o mapa gerado por $K R G$ apresenta um padrão mais adequado à distribuição espacial da chuva. Apesar dos parâmetros estatísticos sugerirem a interpolação por $R B F$, visualmente observa-se um aumento da área representada pela classe de maiores valores, comparados aos demais interpoladores.

Os resíduos gerados pela validação cruzada, a partir da interpolação por $K R G$, sugerem melhor eficiência dos parâmetros para a maior intensidade do dia $27 / 11$. Os dados da média, desvio padrão e $R E Q M$ foram significativamente menores, o que por consequência acarretou nos melhores índices de $r, d$ e $c$. Enquanto os dados interpolados por IDW e RBF apresentaram índice de desempenho sofrível, por $K R G$ classificou-se como bom. Segundo Castro et. al., este método apresenta melhor desempenho para interpolação deste elemento do clima em diversas regiões do País, conforme mostram os trabalhos de Carvalho \& Assad (2005), Barbosa (2006) e Silva et al. (2007), ressaltando, desta forma, os resultados encontrados no presente estudo.

Muitos trabalhos em climatologia utilizam-se do interpolador $I D W$, devido a sua simplicidade de manuseio, como em Reis et. al. (2005), Braz et. al. (2007) e Amorim et. al. (2006). Em todas as 5 amostras, nos três diferentes métodos, a interpolação por IDW foi a menos indicada para geração dos mapas de chuva, tanto em amostras mais homogêneas, como naquelas de forte tendência espacial, o interpolador não apresentou parâmetros estatísticos satisfatórios.

O método RBF é muito pouco citado na literatura como interpolador de chuva, porém apresentou resultados melhores que o IDW e em alguns casos, melhor que a $K R G$. Apesar de ser um método simples, que não necessita de análise variográfica, apresentou desempenho considerável em relação aos demais. Esta analise é fundamental para identificação de valores discrepantes dos vizinhos mais próximos. Em amostras de dados 
mais assimétricas, como a maior intensidade do dia 15, só a análise variográfica permitiu identificar os pontos de amostragem que dificultaram um bom desempenho do método.

Tabela 3 - Parâmetros estatísticos dos resíduos

\begin{tabular}{|c|c|c|c|c|c|c|c|c|}
\hline \multicolumn{2}{|c|}{} & $\begin{array}{c}\text { Média } \\
\text { dos } \\
\text { Resíduos }\end{array}$ & $\begin{array}{c}\text { Desvio } \\
\text { Padrão } \\
\text { dos } \\
\text { Resíduos }\end{array}$ & REQM & $\begin{array}{c}\text { Correlação } \\
(r)\end{array}$ & $\begin{array}{c}\text { Concordância } \\
(d)\end{array}$ & $\begin{array}{c}\text { Confiança } \\
(c)\end{array}$ & Desempenho \\
\hline \multirow{3}{*}{\begin{tabular}{c} 
Mensal \\
\cline { 2 - 9 }
\end{tabular}} & IDW & 3,50 & 31,36 & 13,7 & 0,81 & 0,83 & 0,68 & Bom \\
\cline { 2 - 9 } & RBF & 2,00 & 24,1 & 7,86 & 0,86 & 0,87 & 0,75 & Bom \\
\cline { 2 - 9 } $\begin{array}{c}\text { Dia } \\
15 / 11\end{array}$ & KRG & 2,16 & 22,2 & 3,28 & 0,89 & 0,88 & 0,79 & Muito Bom \\
\cline { 2 - 9 } & RBF & 1,29 & 8,21 & 5,47 & 0,11 & 0,73 & 0,08 & Péssimo \\
\cline { 2 - 9 } & KRG & 1,99 & 7,79 & 4,19 & 0,37 & 0,73 & 0,27 & Péssimo \\
\hline \multirow{2}{*}{$\begin{array}{c}\text { Dia } \\
27 / 11\end{array}$} & IDW & $-1,8$ & 11,1 & 7,60 & 0,86 & 0,85 & 0,73 & Bom \\
\cline { 2 - 9 } & RBF & 0,25 & 9,3 & 1,04 & 0,87 & 0,88 & 0,77 & Muito Bom \\
\cline { 2 - 9 } & KRG & 1,08 & 10,1 & 4,56 & 0,84 & 0,85 & 0,71 & Bom \\
\hline \multirow{2}{*}{$\begin{array}{c}\text { Horária } \\
15 / 11\end{array}$} & IDW & 1,14 & 5,45 & 3,79 & $-0,62$ & 0,56 & $-0,34$ & Péssimo \\
\cline { 2 - 9 } & RBF & 0,48 & 5,73 & 1,61 & $-0,50$ & 0,57 & $-0,28$ & Péssimo \\
\cline { 2 - 9 } & KRG & 0,29 & 7,63 & 0,96 & $-0,44$ & 0,56 & $-0,25$ & Péssimo \\
\hline \multirow{2}{*}{$\begin{array}{c}\text { Horária } \\
27 / 11\end{array}$} & IDW & $-0,86$ & 5,14 & 3,65 & 0,68 & 0,81 & 0,55 & Sofrível \\
\cline { 2 - 9 } & RBF & $-0,29$ & 5,16 & 1,26 & 0,67 & 0,81 & 0,54 & Sofrível \\
\cline { 2 - 9 } & KRG & $-0,12$ & 3,72 & 0,49 & 0,84 & 0,86 & 0,72 & Bom \\
\hline
\end{tabular}

Fonte de dados: URBEL/UFMG e INMET.

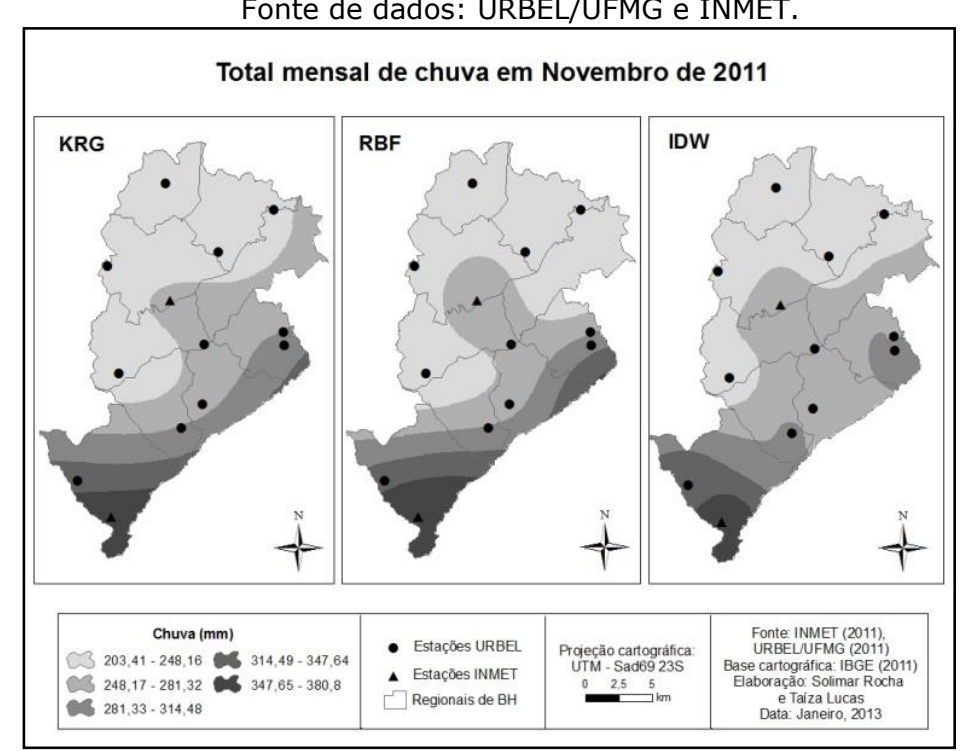

Figura 2: Mapas do total mensal de chuvas de novembro de 2011 interpolados por Krigagem ordinária modelo esférico $(K R G)$, Radial Basis Functions (RBF) e Inverse Distance Weighting (IDW). Fonte de dados: URBEL/UFMG e INMET. 


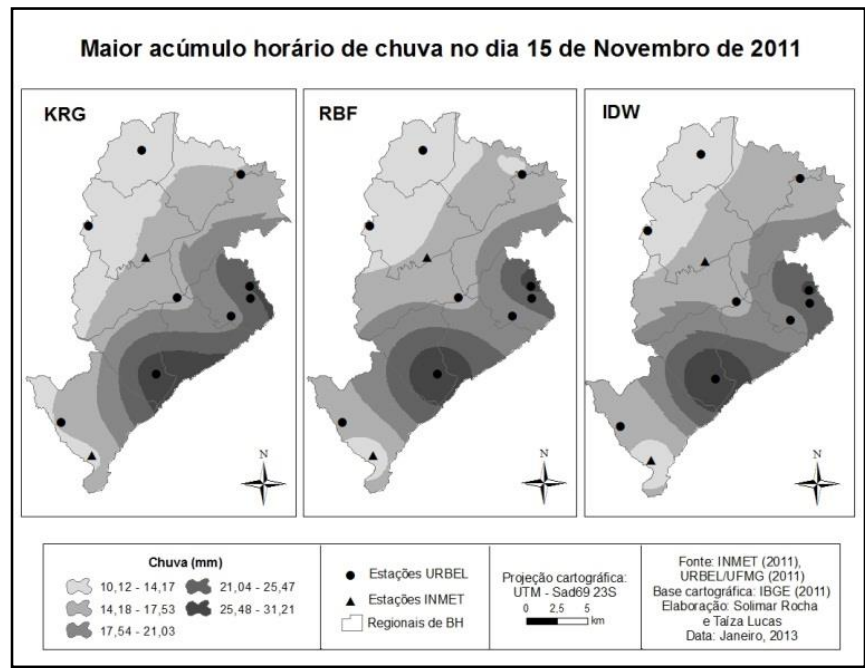

Figura 3: Mapas do total horário de chuvas do dia 15 de novembro de 2011, interpolados por Krigagem ordinária modelo esférico (KRG), Radial Basis Functions (RBF) e Inverse Distance Weighting (IDW). Fonte de dados: URBEL/UFMG e INMET.

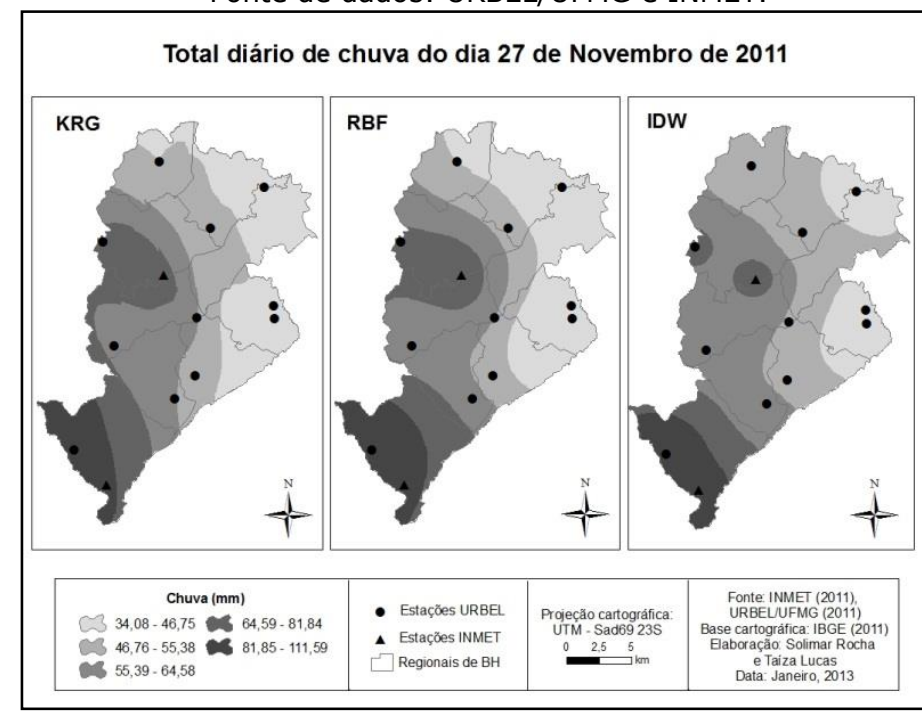

Figura 4: Mapas do total diário de chuvas do dia 27 de novembro de 2011, interpolados por Krigagem ordinária modelo esférico (KRG), Radial Basis Functions (RBF) e Inverse Distance Weighting (IDW). Fonte de dados: URBEL/UFMG e INMET.

\section{CONCLUSÕES}

A utilização de técnicas estatísticas e geoestatística com análise variográfica mostrou que as características estruturais estudadas da precipitação estão correlacionadas e apresentam forte dependência espacial. A análise estatística é uma etapa fundamental para conhecimento da distribuição dos dados, assim como a sua variância. A partir do CV tem-se uma estimativa da qualidade da interpolação, confirmada pelos parâmetros estatísticos dos resíduos produzidos pela validação cruzada. O menor valor de CV foi observado pelos dados mensais de novembro, para o qual todos os interpoladores mostraram os melhores índices de desempenho.

A análise da distribuição dos dados a partir dos gráficos de histograma e QQPlot são importante para entendimento da distribuição da amostra e identificação de dados distribuídos aleatoriamente e outliers. A precipitação é uma variável de difícil ajustamento aos padrões de distribuição normal devido à alta variabilidade espacial, portanto o entendimento da espacialização associada ao conhecimento da área e dos padrões atmosféricos de geração da mesma é fundamental nas escolhas do tratamento da variável. 
A análise de tendência espacial e variográfica são fundamentais para o ajuste dos parâmetros necessários a modelagem, particularmente a KRG. É necessário rever os gráficos QQPlot e compará-los aos gráficos de semivariograma, covariância e os pares de dados distoantes das amostras a partir do comportamento entre os vizinhos, em função da distância. Os mapas da figura 3 apresentaram um padrão espacial de ocorrência da chuva horária (SE-NW) concentrada nas franjas da Serra do Curral, na Regional CentroSul e suas proximidades, identificado a partir de sucessivas análises variográficas, no qual o melhor ajuste foi encontrado com a retirada de três postos distoantes da distribuição normal, indicados pelo gráfico QQPlot.

A validação cruzada é um procedimento fundamental para geração dos resíduos e aplicação dos parâmetros estatísticos necessários ao entendimento da qualidade da interpolação, só a análise visual dos mapas não é suficiente para definir os métodos de interpolação. Os parâmetros estatísticos de tendência central e variância, como a média dos resíduos, desvio padrão e REQM são fundamentais, principalmente na ausência ou baixa correlação entre os dados estimados e observados. O índice de correlação $(r)$ indica o grau de dispersão dos dados obtidos em relação à média. O índice $d$ indica o grau de afastamento dos valores estimados em relação aos observados. Seus valores variam de zero, para nenhuma concordância, a 1, para a concordância perfeita. Quando os dados estão muito afastados da média, o índice $d$ é extremamente relevante para identificação da qualidade do interpolador.

$O$ índice de confiança $c$ qualificou a $K R G$ como o melhor interpolador de três amostras, das cinco estudadas. O método requer análise variográfica, assim como ajuste aos modelos mais adequados de semivariograma, o que é uma vantagem em relação aos interpoladores determinísticos. É necessário analisar os parâmetros do variograma (efeito pepita, alcance, patamar e número de estruturas presentes) até que se considere o modelo satisfatório à distribuição dos dados. A $K R G$ será o interpolador utilizado em estudos de espacialização da chuva em Belo Horizonte, no intuito de entender o padrão espacial dos tipos de chuva, devido as suas possibilidades de modelagem, o que permite a geração de mapas mais consistentes, com homogeneidade entre os vizinhos mais próximos e sem "ilhas" em torno dos valores extremos.

Muitos trabalhos em climatologia utilizam-se do interpolador IDW, porém os resultados desta pesquisa mostram que além dos problemas visuais, geração de ilhas concêntricas, apresenta menor qualidade estatística na estimativa. A análise visual é também um processo necessário na escolha dos melhores métodos, o RBF apresenta linhas de isoietas mais suavizadas, mas tende a aumentar as áreas de influência dos maiores e menores valores. Apesar de ter sido o melhor interpolador para os dados diários a partir da análise estatística dos resíduos.

\section{REFERÊNCIAS BIBLIOGRÁFICAS:}

AMORIM, F. C. F, RIBEIRO, A. LEITE, C. C, LEAL, B. \& SILVA, J. B. da. Avaliação de dois métodos de espacialização da precipitação pluvial para o estado de Alagoas. XIV Congresso Brasileiro de Meteorologia. Florianópolis, 2006. Anais eletrônicos. CD-ROM. Florianópolis: SBMET, 2006.

ANDRIOTTI, J. L. S. Notas de Geoestatística. Revista Acta Geologica Leopoldensia. XXV (55): 3 $-14,2002$.

ANDRIOTTI, J. L. S. Fundamentos de estatística e geoestatística. São Leopoldo: Ed. Unisinos, 2003. $165 p$.

ARANOFF, S. Geographic information systems: a management perspective. Ottawa: WDL Publications, 1989. 294p.

BARBOSA, J. P. M. Utilização de método de interpolação para análise e espacialização de dados climáticos: O Sig como ferramenta. Caminhos da Geografia, v.9, n.17, p.85-96, 2006.

BRAZ, R. L, RIBEIRO, C. A. D, FERREIRA, D. S. \& CECILIO, R. A. Uso de séries históricas e técnicas de SIG no estudo da distribuição temporal e espacial da pluviosidade na bacia Barra Seca localizada ao norte do Estado do Espírito Santo. XI Encontro Latino Americano de Iniciação Científica, 2007, São José dos Campos. Anais. 2007. Vol. 14. 54-57p. 
CAMARGO, A. P. \& SENTElHAS, P. C. Avaliação do desempenho de diferentes métodos de estimativa da evapotranspiração potencial no estado de São Paulo, Brasil. Revista Brasileira de Agrometeorologia. v.5, n.1, p,89-97, 1997.

CARVALHO, J. R. P.; ASSAD, E. D. Análise espacial da precipitação pluviométrica no estado de São Paulo: comparação de métodos de interpolação. Engenharia Agrícola, v.25, n.2, p.377-384, 2005.

CAStro, F. da S. PeZzopane, J. E. M, CECÍlio, R. A, PEZZOPANE, J. R.M. \& XAViER, A. C. Avaliação do desempenho dos diferentes métodos de interpoladores para parâmetros do balanço hídrico climatológico. Revista Brasileira de Eng. Agrícola e Ambiental. Campina Grande. UAEA/UFCG. Março de 2010.

ESRI. Arcgis 9: Using Arcgis Geostatistical Analyst. Nova Iorque, 2003. 306p.

LANDIM, P. M. B. Sobre geoestatística e mapas, Revista Terra Didática, Campinas, v. 2, n. 1, p,19-33, 2006.

MARCUZZO, F. N, ANDRADE, L. R. \& MELO, D. C. R. Métodos de Interpolação Matemática no Mapeamento de Chuvas do Estado do Mato Grosso. Revista Brasileira de Geografia Física. Vol. 4. Ano 2011. 793-804p.

MOREIRA, J. L. B. Estudo da distribuição espacial das chuvas em Belo Horizonte e em seu entorno. 2002. 186f. Dissertação (Mestrado em Geografia e Análise Ambiental): Instituto de Geociências, Universidade Federal de Minas Gerais, Belo Horizonte, 2002.

MOURA, A. C. M. A escolha de interpoladores e recursos de visualização na estruturação de bases de dados para produção de informações espaciais apoiadas por geoprocessamento. Laboratório de Geoprocessamento da Escola de Arquitetura da UFMG. Belo Horizonte, 2012. 21 p.<http://www.arq.ufmg.br/SiteLabGeo>.

ROGRERSON, P. A. Métodos estatísticos para geografia. Porto Alegre: Bookman, 2012. 348p.

REIS, M. H, GRIEBELER, N. P, SARMENTO, P. H. L, OLIVEIRA, L. H, OLIVEIRA, J. M. Espacialização de dados de precipitação e avaliação de interpoladores para projetos de drenagem agrícola no estado de Goiás e Distrito Federal. Anais. XII Simpósio Brasileiro de Sensoriamento Remoto. Goiânia, 16-21 de abril de 2005.

REIS R. J, GUimARÃES, D. P, COELHO, C. W. G. A, PAIXÃO, G. M. M, NASCIMENTO, J. S, SIMÕES, T. K. S. L. Determinação das áreas de potencial de riscos de precipitações intensas em Belo Horizonte. XIII Congresso Brasileiro de Meteorologia. 2004. Fortaleza, Anais. Ceará: 2004.

SILVA, J. MONTEIRO, P. NEGREIROS, J. AGUILAR, F. AGUILAR, M. Modelação Espacial da Precipitação na Ilha de Santiago, Cabo Verde, com GeoStatistical Analyst. Livro de Actas Iask. Cabo Verde, 2011.

SILVA, K. R.; PAIVA, Y. G.; CECÍLIO, R. A.; PEZZOPANE, J. E. M. Avaliação de interpoladores para a espacialização de variáveis climáticas na bacia do rio Itapemirim-ES. Simpósio Brasileiro de Sensoriamento Remoto, 13, 2007, Florianópolis. Anais. São José dos Campos: INPE, 2007. p.3141-3146.

TAO, T, CHOCAT, B, LIU, S. \& XIN, K. Uncertainty analysis of interpolation methods in rainfall spatial distribution - a case of small catchment in Lyon. Journal Water Resource and Protection. USA. $\mathrm{n}^{\circ}$ 2, p, 136-144. 2009.

WANDERLEY, H. S, AMORIM, R. F. C. \& CARVALHO, F. O. Variabilidade espacial e preenchimento de falhas de dados pluviométricos para o Estado de Alagoas. Revista Brasileira de Meteorologia. v.27, n³, p,347 - 354, 2012.

WILLMOTT, C. J. CKLESON, S. G. DAVIS, R. E. Statistics for the evaluation and comparison of models. Journal of Geophysical Research. Ottawa, v. 90, n. C5, p.8995-9005, 1985. 